\title{
A further look at social desirability
}

\author{
MIRIAM RODIN \\ California State University, San Diego*, Calif. 92115
}

The pervasiveness of social-desirability (SD) responding on self-report instruments has been apparently well demonstrated. It is suggested here that the generality of this finding may be partly an artifactual result of the methodology used to demonstrate it. Studies directed to this question happen to have used self-report instruments with two characteristics in common: personalityadjustment content and two-choice formats. Both of these characteristics would be expected to heighten SD responding: adjustment items because they are explicitly coded for SD in the culture, and two-choice formats because Ss cannot readily disclaim the (higher) $\mathrm{SD}$ alternative without incriminating themselves. The instrument used here differed in both respects. The item format was multiple choice, and the item contents were situationally defined behaviors shown to be not explicitly coded for SD in the culture. Ss were asked to make "typical," SD, and actual behavior judgments for each of the items and to indicate their confidence in the judgments. The findings were replicated with independent sets of both Ss and items. It was concluded that previous work has overestimated the extent of SD responding over self-report instruments in general. The present items may offer a promising direction for constructing self-report instruments less likely to be affected by the SD set.

It seems to have been clearly established that responses to personality inventories can be fairly well predicted by the social desirability (SD) values of the items (Edwards, 1953, 1957). ${ }^{1}$ However, most SD studies have been based on items (typified ky the MMPI) that have in common both a content and format characteristic. The item contents have referred to inner states of being and feeling, and have been more or less explicitly tied to "adjustment." The basic format has been two-choice: endorse one of two alternatives or agree/disagree with the one given. Both these characteristics might be expected to heighten SD responding: adjustment content because it is fairly explicitly coded for SD in the culture, and two-choice formats because Ss cannot disclaim the SD alternative without incriminating themselves. Instruments possessing these characteristics would thus be expected to yield an inflated estimate of the extent of SD responding over self-report instruments in general. The items used in the present study differ in both content and format from the preceding.

The item contents are behavior statements. They refer not to inner states but to specific actions in a well-defined situational context. It is expected that behavior statements are less explicitly coded for $\mathrm{SD}$ in the culture than are "adjustment" items (an assumption examined in the course of the study) and therefore exert less pressure on Ss to claim the maximum social desirability embedded in the item.

\footnotetext{
*Formerly San Diego State College.
}

The item format is multiple choice. This format has two advantages. First, it unconfounds acquiescence and social desirability. Previous attempts at keying personality inventories to make them immune to the SD set have been plagued by an unfortunate ambiguity; for an item phrased in a positive direction, to which a "yes" answer is also the socially desirable answer, it is moot whether the response reflects acquiescence bias, social desirability bias, or the simple truth about the individual. Multiple choice at least eliminates acquiescence from the bundle. The second advantage of this format is that it allows the $\mathbf{S}$ to deny the maximum social desirability embodied in the question without incriminating himself. In the two-valued response format, the alternatives are perforce "most" and "least" desirable, and the $S$ cannot deny social desirability without admitting at the same time to being socially undesirable. The strategy of attempting to equate response alternatives for social desirability fails because the forced choice format actually heightens the S's ability to discriminate between them on the SD dimension (Feldman \& Corah, 1960). The $S$ is not much helped by the middle or "?" category since its use is usually actively discouraged by the $\mathrm{E}$. (It is a common practice to discard as invalid the protocols of Ss who persist in using the middle category extensively.)

In short, the first object of this study was to look at the extent to which Ss claim SD on a self-report instrument different in both format and content from those usually used. The hypothesis is that on such an instrument Ss will more often describe themselves by statements other than those they judge to be most socially desirable. This will occur because (1) the multiple choice format permits response options other than most and least desirable, and (2) the "behavior" content of the items, relative to " adjustment" content, is weakly coded by the culture for social desirability. The degree of cultural explicitness with respect to the social desirability of these behaviors is estimated here by the extent of $S$ consensus on SD judgments.

\section{METHOD}

The basic procedure was straightforward. Ss were presented with a self-report instrument composed of 11 behavior items. Each item was a fairly specific description of a social situation, along with a set of four alternative responses possible in that situation. The Ss were asked to make four judgments about each item. For each judgment, Ss were asked to give a confidence rating of 1 (positive), 2 (reasonably certain), or 3 (uncertain).

Two different self-report instruments were used: $S_{A}$ was administered to $21 \mathrm{Ss}, \mathrm{SR}_{\mathrm{B}}$ to $24 \mathrm{Ss}$. Because both the items and the subjects were different, the data collected with $S R_{A}$ and $S R_{B}$ constitute independent replications. This is important to note, because results consistent over both sets of data argue strongly that it is the type of item, rather than the particular set of experimental items, that is the operative variable.

An example of an item and the judgment questions (which were the same for all items) is:

C.T. asks $\mathrm{J}$ to lend him $\$ 5.00$. He knows $J$ just got paid that morning and has plenty of money. $J$ refuses by saying he can't afford the loan right now. C.T. could :

(a) let $J$ know that he is disappointed.

(b) change the subject and go on talking as though nothing has happened.

(c) ask $J$ why he doesn't have enough money since he just got paid that morning.

(d) try to talk him into it.

(1) What would you be most likely to do in this situation?

(2) What do you think most people would really do in this situation?

(3) What is the most socially desirable thing to do in this situation?

(4) What is the least socially desirable thing to do in this situation?

Within each set, there was a further division into two subsets. For each of the 11 items in a set, Ss were asked to 
Judgment

\begin{tabular}{|c|c|c|c|c|}
\hline & \\
\hline & $\left(\begin{array}{l}1 \\
\text { Do }\end{array}\right.$ & $\begin{array}{c}2 \\
\text { (Typical) }\end{array}$ & $\begin{array}{c}3 \\
(S D)\end{array}$ & $\begin{array}{c}4 \\
\text { (UD) }\end{array}$ \\
\hline $\begin{array}{c}\text { Overall Mean } \\
\text { A } \\
\text { B }\end{array}$ & $\begin{array}{l}.52 \\
.55 \\
.49\end{array}$ & $\begin{array}{l}.40 \\
.41 \\
.38\end{array}$ & $\begin{array}{l}.59 \\
.63 \\
.56\end{array}$ & $\begin{array}{l}.62 \\
.65 \\
.58\end{array}$ \\
\hline Confidence Intervalt & $.47-.57$ & $.36-.44$ & $.55-.64$ & $.56-.68$ \\
\hline
\end{tabular}

*By chi-square, if the consensus score is $\geqslant .35$ in Set $A$ or $\geqslant .32$ in Set $B$, then $p<.05$ that the consensus is random.

tBased on combined $N$ since there was homogeneity of variance.

respond under two different conditions. The conditions were defined by the relationship of the other in the situation to the central actor. With reference to the example above, $J$ is a close friend of C.T. in Subset 1 ; he is a casual acquaintance in Subset 2. The difference in relationship varied over items and included such pairs as teacher vs peer, someone liked vs not liked, etc. There was little difference between subset conditions. They are used to check reliability but are combined in the rest of the data analysis.

\section{Scoring}

The dependent variables are two kinds of scores: consensus scores and pairing scores. Consensus scores are a linear measure of agreement among judges. They were obtained for every judgment for every item. Consensus scores vary from 0 to 1,0 indicating a perfectly random scatter of answers to a particular question, and 1 indicating perfect interjudge agreement on a single answer. The consensus (C) score is essentially a ratio of observed agreement to perfect agreement. It is related to chi square

$$
C^{2}=\frac{x^{2}}{(k-1)(N)}
$$

and hence may be evaluated on a known sampling distribution. The advantage of $\mathrm{C}$ scores over chi square is their linearity: one may conclude that there is twice as much interjudge agreement on an item with $\mathrm{C}=.6$ than on an item with $\mathrm{C}=.3$. Pairing scores are a within-judge measure. They are the proportion of items on which judges select the same alternative to describe both the socially desirable behavior and their own behavior. The pairing scores were normalized by an arcsin transformation. The confidence limits were calculated from the transformed scores.

\section{RESULTS}

Ss ascribed socially desirable behaviors to themselves $\mathrm{X}=58 \%$ of the time $\left(\overline{\mathrm{X}}_{\mathrm{A}}=.62, \overline{\mathrm{X}}_{\mathrm{B}}=.55\right)$. The $95 \%$ confidence limits, based on a combined $\mathrm{N}$ since there was homogeneity of variance, are $.53<\mathrm{p}_{\mathrm{o}}$ $<.63$. This result is not directly comparable with data presented in terms of correlations between social desirahility and likelihood of endorsement. Nevertheless, the proportion (.42) of responses in which Ss described themselves in terms of behavior other than those they rated as most socially desirable seems fairly substantial relative to the presumed pervasiveness of the tendency to claim social desirability. It should be noted that discrepancies between "self-descriptive" and "socially desirable" responses cannot be attributed to individual differences in the conception of what is socially desirable since the results are based on intra-individual scores. This is not the case with independent SD keying: when a S's response differs from the keyed response, it is not clear whether he has chosen the "undesirable" response or whether he simply has a different view of which is the "desirable" response (Scott, 1963; Wiggins, 1966).

It might be argued that some items are harder to judge, or less conventionally phrased, or whatever, and it is these items that account for the obtained disagreements between self-descriptive and socially desirable responses. However, an $F$ ratio of less than 1 for item effects in the number of pairings would seem to indicate that items did not reliably differ from one another in the extent to which they led Ss to pair responses.

$$
\text { Consensus }
$$

Table 1 substantiates the assumption that the item contents are weakly coded for SD in the culture. Consensus on SD judgments (Questions 3 and 4) was not significantly greater than consensus on actual behavior judgments (Question 1). Consensus on actual behavior is a good baseline for evaluating the lower limit of homogeneity in the sample. Maximum variability would be expected on judgments of actual behavior and maximum agreement on judgments of ideal behavior. This discrepancy should obtain unless Ss (quasi-) always describe their behavior as SD, or there is not a well-defined cultural ideal with respect to the social desirability of the behaviors. The latter explanation would seem to be the correct one since the former is ruled out by the previous finding that Ss are capable of making quite clear distinctions between their behavior and the one they judge to be most socially desirable. Of incidental interest is the suggestion that cultural sanctions may be clearer on what one should not do than on what one should: consensus on "social undesirability" judgments was consistently (although not significantly) higher than consensus on "social desirability" judgments.

The similarity in consensus on actual and ideal behavior is not an artifactual outcome of the fact that the items differ greatly from one another in the extent to which they encourage consensus. That is, it could be said that some of the situations lead to clear-cut decisions on what one does and ought to do, and on these items consensus on both judgments will be high; whereas other items are more ambiguous and hence consensus on both questions will be correspondingly lower. This explanation is not supported by the data. The correlation of consensus scores on Questions 1 and 3 is essentially zero (-.02). In short, consensus scores on the various judgment questions are not tied to one another, and the rolation between them cannot be explained on the basis of item characteristics.

Reliability

Is the lack of high consensus among judges the result of a process akin to guessing? The answer is negative if one is willing to ascribe face validity to the confidence ratings. Overall, Ss claimed to be very confident in all their ratings. Even on Question 2, which has the lowest confidence rating, Ss are slightly more confident than "reasonably certain." One can question the meaningfulness of the confidence ratings on the grounds that they are crowded at the upper end of the scale. Evidence that the ratings are nevertheless meaningful is that they do discriminate between judgments and in the expected ways. For both groups, $S R_{A}$ and $S_{B}$, ANOVA indicates that Ss are significantly more confident in the judgment of their own behavior than in the other judgments $(p<.001)$ and significantly less confident when judging what most people will do than when judging what they ought to do $(p<.001)$.

Another index of response reliability is given by the proportion of change in judgments to the same items between subsets. This measure probably underestimates reliability 
relative to a standard test-retest measure because the identity of the other person present in the stimulus situation differed across subsets and this variable would be expected to change judgments to some extent. The judgment least likely to be affected by the identity of the other person should be the social undesirability judgments (Question 4): for these, the mean proportion of changes across subsets was minimal $\left(\overline{\mathrm{X}}=.12 ; \mathrm{p}_{95} .09<\mathrm{p}_{\mathrm{o}}<\right.$ $.16)$.

\section{DISCUSSION}

It was found that Ss described their behavior as different from the behavior they judged to be most socially desirable a substantial proportion of the time. The self-report instrument used in this study differed from earlier ones in both item form and item content. It cannot be determined whether the present outcome is due to the specific behavioral content of the items, to the inclusion of several acceptable alternatives in the item format, or to some combination of these two factors. In any case, it seems clear that previous work has yielded an inflated estimate of the extent of SD responding over self-report instruments in general.

Since greatest variability would be expected in Ss' descriptions of their actual behavior, consensus on this question establishes the limit of heterogeneity in the particular experimental sample. It was found that consensus among judges on the most socially desirable response was not significantly greater than consensus on actual behavior. Ss were about as idiosyncratic in judgments of what one ought to do as in judgments of what they actually would do. It is clearly undesirable to be maladjusted or to lack moral rectitude. For specific situationally bound behaviors, however, societal sanctions on social desirability are apparently far less clear.

It may be concluded that it is possible to construct self-report instruments that at least reduce considerably the extent to which Ss' responses are predictable by independent social desirability keying. The choice of behavioral exemplars for dimensions of interest seems to remove the clarity and hence some of the force of cultural definitions of social desirability so that Ss feel freer not to claim it. Furthermore, it is far easier to disclaim maximum social desirability when one can still claim some social desirability - a choice that is apparently not subjectively present for those who must choose between only two alternatives.

\section{REFERENCES}

CROWNE, D $P$ \& MARLOWE, D. A new scale of social desirability independent of psychopathology. Journal of Consulting Psychology, 1960, 24, 349-354.

DAMARIN, F., \& MESSICK, S. Response styles as personality variables: A theoretical integration of multivariate research. Research Bulletin 65-10. Princeton, N.J: Educational Testing Service, 1965.

EDWARDS, A. L. The relationship between the judged desirability of a trait and the probability that the trait will be endorsed. Journal of Applied Psychology, $1953,37,90-93$.

EDWARDS, A. L. The social desirability variable in personality assessment and research. New York: Dryden, 1957.

FELDMAN, M., \& CORAH, N. Social desirability and the forced-choice method. Journal of Consulting Psychology, 1960, 24, 480-482.

SCOTT, W. A. Social desirability and individual conceptions of the desirable. Journal of Abnormal \& Social Journal of Abnormal \&

WIGGINS, N. Individual viewpoints of social desirability. Psychological Bulletin, $1966,66,68-77$.

\section{NOTE}

1. This comment does not apply to scales composed of items with an a priori low expected frequency of endorsement, such as the Marlowe-Crowne (1960). Such items seem to tap a separate dimension (Damarin \& Messick, 1965). 